Letter

\section{Peripheral neuropathy in ALS: phenotype association}

\section{INTRODUCTION}

Amyotrophic lateral sclerosis (ALS) is a rare and progressive neurodegenerative disease mainly affecting upper and lower motor neurons but also causing multisystem involvement, in particular, associated with cognitive changes. Minor sensory fibre dysfunction has been described in the past $^{1}$ and confirmed in recent studies. ${ }^{2}$ In a multicentre study investigating a population of 88 patients with ALS, the ESTEEM group (a European Telematic Project for quality assurance within Clinical Neurophysiology) reported sensory polyneuropathy (PNP) in $12.5 \%$ of the patients, not influenced by age, disease duration and onset region.

In this study, we aimed to readdress prevalence of and risk factors for PNP in a larger population of patients with ALS. A large number of variables, including gene mutations, were assessed.

\section{METHODS}

We prospectively followed up patients with ALS in Lisbon (January 2015January 2018) consecutively enrolled into the OnWebDuals register ${ }^{3}$ to test the influence of clinical features and genotype on PNP prevalence. We included patients older than 18, with possible, probable or definite ALS according to the revised El Escorial criteria, and in line with the Awaji electrophysiological guidelines. Patients with known PNP, marked lower limb oedema, monoclonal gammopathy and those with incomplete neurophysiological examination were excluded. Age, gender, onset region, disease duration, weight loss before diagnosis (>10\%), history of diabetes or cancer, previous chemotherapy or contact with neurotoxic agents, as well as SOD1 and C9orf72 mutations were recorded. Disease duration was defined as the time between the onset of muscle weakness and the neurophysiological investigation, which was performed at diagnosis or during confirmatory evaluation for patients referred from other centres.

The study cohort underwent standardised nerve conduction studies as part of the assessment protocol. Motor conduction studies included bilateral peroneal and right ulnar nerves (distal latency and velocities), including F-waves (latency and persistence). Sensory action nerve potentials (SNAPs) were recorded from peroneal nerves (distal leg, dorsum pedis) bilaterally (conduction velocity and amplitude), if one or both were abnormal additional SNAPs from sural nerves were recorded bilaterally (sura, behind the lateral malleolus). Stimulation and recording were performed through surface electrodes, and skin temperature was kept above $30^{\circ} \mathrm{C}$. SNAP amplitude $<$ mean $+2.5 \mathrm{SD}$ (compared with age and gender-matched subjects from a historical control group) was accepted as abnormal. PNP was defined by the presence of one or two abnormal SNAPs from the peroneal nerves plus one or two abnormal SNAPs from the sural nerves. As established by Awaji guidelines, the presence of a mild PNP should not exclude the diagnosis of ALS, taking into account the clinical presentation and the full set of neurophysiological abnormalities. ${ }^{4}$ Patients with ALS with neurophysiological signs of neuropathy were grouped in group 2 (G2) and the remaining in group 1 (G1).

Mann-Whitney $U$ test and $\chi^{2}$ or Fisher exact test were used to study differences between groups for continuous and categorical variables, respectively. Logistic regression analysis was applied to identify independent predictors for PNP (binary dependent variable). A $\mathrm{p}$ value of $<0.05$ was considered as significant.

\section{RESULTS}

We included 339 patients with ALS, 191 men (56.3\%), with spinal $(n=243$,

Table 1 Demographic characteristics of the total population and of both groups (G1, no PNP and G2, with PNP)

\begin{tabular}{|c|c|c|c|c|}
\hline & Population & G1 (no PNP) & G2 (with PNP) & P value* \\
\hline Number of patients & 339 & $310(91.4 \%)$ & $29(8.6 \%)$ & \\
\hline Gender (men), n (\%) & $191(56.3)$ & $169(54.5)$ & $22(75.9)$ & $0.027^{*}$ \\
\hline Onset age (years) & $61.54 \pm 13.5$ & $61.00 \pm 13.5$ & $67.31 \pm 13.3$ & $0.01^{*}$ \\
\hline Disease duration (months) & $21.03 \pm 30.6$ & $21.18 \pm 31.3$ & $19.47 \pm 20.9$ & 0.86 \\
\hline \multicolumn{5}{|l|}{ Onset form, n (\%) } \\
\hline Spinal & $243(71.7)$ & $223(71.9)$ & $20(69.0)$ & 0.83 \\
\hline Bulbar & $75(22.1)$ & $70(22.6)$ & $5(17.2)$ & 0.64 \\
\hline Respiratory & $8(2.4)$ & $5(1.6)$ & $3(10.3)$ & $0.024^{*}$ \\
\hline Axial & $6(1.8)$ & $5(1.6)$ & $1(3.4)$ & 0.418 \\
\hline Dyscognition & $5(1.5)$ & $5(1.6)$ & 0 & 1 \\
\hline Generalised & $2(0.6)$ & $2(0.6)$ & 0 & 1 \\
\hline Weight loss (yes), n (\%) & $53(15.6)$ & $51(16.5)$ & $2(6.9)$ & 0.28 \\
\hline Diabetes (yes), n (\%) & $39(11.5)$ & $35(11.3)$ & $4(13.8)$ & 0.76 \\
\hline Cancer (yes), n (\%) & $32(9.4)$ & $28(9)$ & $4(13.8)$ & 0.34 \\
\hline Chemotherapy and/or neurotoxic (yes), n (\%) & $8(2.4)$ & $7(2.3)$ & $1(3.4)$ & 0.52 \\
\hline SOD1 (yes), n (\%) & $2 / 130$ & $2 / 119$ & $0 / 11$ & 1 \\
\hline C9orf72 (yes), n (\%) & $18 / 233$ & $17 / 214$ & $1 / 19$ & 1 \\
\hline
\end{tabular}

P significant for $<0.05$.

ALS, amyotrophic lateral sclerosis; PNP, polyneuropathy; SNAPs, sensory action nerve potentials.

$71.7 \%)$, bulbar $(\mathrm{n}=75,22.1 \%)$, respiratory $(n=8,2.4 \%)$, axial $(n=6,1.8 \%)$, cognitive $(n=5,1.5 \%)$ and generalised onset $(n=2,0.6 \%)$. Clinical and neurophysiological data were complete for every patient, while SOD1 and C9Orf72 mutations were tested in 130 and 233 patients, respectively.

From the total study population, 29 patients had PNP according to the definition described earlier $(8.6 \%, \mathrm{G} 2)$ vs 310 patients without $(91.4 \%, \mathrm{G} 1)$. SNAPs in all patients in G2 showed with mild or moderate amplitude reduction; sensory conduction velocity was borderline in 19 patients and slightly reduced in 10 . No patient was symptomatic for the neuropathy.

All demographic variables (table 1) were similar between groups $(p>0.05)$ except for gender, age and frequency of respiratory onset. G2 was characterised by a higher percentage of men (54.5\% G1 vs 75.9\% G2, p=0.027), older age $(61.00 \pm 13.5 \mathrm{G} 1$ vs $67.31 \pm 13.3 \mathrm{G} 2$, $\mathrm{p}=0.01)$ and more frequent respiratory onset $(\mathrm{p}=0.024)$.

Logistic regression, including the all the variables defined in the Methods section, confirmed that age $(p=0.014)$, gender $(p=0.024)$ and respiratory onset $(p=0.037)$ were independent predictors for PNP. Regarding age, a single additional year increased the risk of PNP by 1.044 (CI 1.009 to 1.08); female gender decreased the risk of PNP by 0.644 (CI 0.145 to 0.874 ); and respiratory onset increased the risk of PNP by 5.4 (CI 1.108 to 26.156).

Neurol Neurosurg Psychiatry October 2021 Vol 92 No 10 


\section{DISCUSSION}

PNP was found in $8.6 \%$ of our patients with ALS, similarly to previous publications. ${ }^{5}$ The slightly lower rate observed in our study can derive from not accepting a slower conduction velocity with normal SNAP amplitude as a marker of PNP, since patients with ALS tend to have cold extremities, and it is not possible to assure normal velocity of the action potential propagation along the axon by normalising skin temperature.

History of diabetes, weight loss at diagnosis, cancer, chemotherapy or SOD1 and C9orf72 HRE mutations were not associated with PNP. However, PNP was more frequent in older men and, most strikingly, in patients with respiratory symptom onset. Many possible reasons underlying this association can be hypothesised, namely, hypercatabolic status and nutritional factors, as well as PNP being part of this specific phenotype. We speculate that uncompensated peripheral hypoxia could cause peripheral nerve injury in this group of patients, as reported in diabetes, respiratory diseases and critical illness neuropathy.

Patients with respiratory onset were immediately adapted to non-invasive ventilation (NIV). One limitation of our study is that we did not perform follow-up measurements to assess if this intervention modified nerve conduction.

Further limitations of our study are the small number of patients presenting with respiratory onset, lack of genetic testing in some patients and of detailed information about the nutritional status and neurophysiological follow-up examinations. We present, however, the most complete evaluation of risk factors for PNP in ALS, specifically by including genetic findings and precise phenotypical characterisation, together with standardised neurophysiological data.

We conclude that older men with respiratory-onset ALS have a higher risk of PNP. Follow-up studies of these patients on NIV would be necessary to understand the possible role of hypoxia in causing peripheral nerve dysfunction.

\section{Mamede deCarvalho $\odot,{ }^{1}$ Marta Gromicho $\odot{ }^{2}$, Peter Andersen $\odot{ }^{3}$ Julian Grosskreutz $\odot{ }^{4}$, Magdalena Kuzma-Kozakiewicz, ${ }^{5}$ Susanne Petri, ${ }^{6}$ Hilmi Uysal, ${ }^{7}$ Susana Pinto ${ }^{1}$}

${ }^{1}$ Faculdade de Medicina-Instituto de Medicina Molecular, Universidade de Lisboa. Centro Hospitalar Universitário Lisboa Norte, Lisboa, Portugal

${ }^{2}$ Instituto de Medicina Molecular, Universidade de Lisboa, Lisboa, Portugal

${ }^{3}$ Department of Pharmacology and Clinical Neuroscience, Umea Universitet, Umea, Sweden ${ }^{4}$ Department of Neurology, Friedrich-Schiller-Universitat Jena, Jena, Thüringen, Germany

${ }^{5}$ Department of Neurology, Medical University of Warsaw, Warszawa, Poland

${ }^{6}$ Department of Neurology, Medizinische Hochschule Hannover, Hannover, Niedersachsen, Germany

${ }^{7}$ Department of Neurology. Faculty of Medicine, Akdeniz University, Antalya, Turkey

Correspondence to Professor Mamede deCarvalho, Faculdade de Medicina-Instituto de Medicina Molecular, Universidade de Lisboa, Lisboa 1649-028, Portugal; mamedemg@mail.telepac.pt

Contributors MdC designed the study methods and wrote the first draft of the manuscript. SPi and MG contributed to the data analysis. PA did some genetic tests. SPi, MK-K, JG, SPe and HU contributed to the discussion and reviewed and modified the manuscript.

Funding This is an EU Joint Programme -

Neurodegenerative Disease Research (JPND) project. The project is supported through national funding organisations under the aegis of JPND (https://www. neurodegenerationresearch.eu/).

Competing interests None declared.

Patient consent for publication Not required.
Ethics approval The study was approved by the local ethics committee and all patients gave informed consent forms for data inclusion.

Provenance and peer review Not commissioned; externally peer reviewed.

(c) Author(s) (or their employer(s)) 2021. No commercial re-use. See rights and permissions. Published by BMJ.

\section{Check for updates}

To cite deCarvalho $\mathrm{M}$, Gromicho $\mathrm{M}$, Andersen $\mathrm{P}$, et al. J Neurol Neurosurg Psychiatry 2021;92:1133-1134.

Received 16 September 2020

Revised 19 November 2020

Accepted 23 November 2020

Published Online First 28 December 2020

J Neurol Neurosurg Psychiatry 2021;92:1133-1134. doi:10.1136/jnnp-2020-325164

\section{ORCID iDs}

Mamede deCarvalho http://orcid.org/0000-0001-75560158

Marta Gromicho http://orcid.org/0000-0003-2111-

4579

Peter Andersen http://orcid.org/0000-0003-0094-5429

Julian Grosskreutz http://orcid.org/0000-0001-9525-

1424

Susana Pinto http://orcid.org/0000-0002-0727-5897

\section{REFERENCES}

1 Shefner JM, Tyler HR, Krarup C. Abnormalities in the sensory action potential in patients with amyotrophic lateral sclerosis. Muscle Nerve 1991;14:1242-6.

2 Pugdahl K, Fuglsang-Frederiksen A, Johnsen B, et al. A prospective multicentre study on sural nerve action potentials in ALS. Clin Neurophysiol 2008;119:1106-10.

3 De Carvalho M, Ryczkowski A, Andersen P, et al. International survey of ALS experts about critical questions for assessing patients with ALS. Amyotroph Lateral Scler Frontotemporal Degener 2017;18:505-10.

4 de Carvalho M, Dengler R, Eisen A, et al. Electrodiagnostic criteria for diagnosis of ALS. Clin Neurophysiol 2008;119:497-503.

5 Pugdahl K, Fuglsang-Frederiksen A, de Carvalho M, et al. Generalised sensory system abnormalities in amyotrophic lateral sclerosis: a European multicentre study. J Neurol Neurosurg Psychiatry 2007;78:746-9. 